\title{
Dynamic Adaptation of Joint Transmission Power and Contention Window in VANET
}

\author{
${ }^{1}$ Danda B. Rawat, ${ }^{2}$ Gongjun Yan, ${ }^{1}$ Dimitrie C. Popescu, ${ }^{2}$ Michele C. Weigle, ${ }^{2}$ Stephan Olariu \\ ${ }^{1}$ Department of Electrical and Computer Engineering, ${ }^{2}$ Department of Computer Science \\ Old Dominion University \\ Norfolk, VA 23529, USA \\ Email: ${ }^{1}\left\{\right.$ drawa001, dpopescu\}@odu.edu, ${ }^{2}$ \{ygongjun, mweigle, olariu\}@cs.odu.edu
}

\begin{abstract}
In this paper, we propose an algorithm for joint adaptation of transmission power and contention window to improve the performance of vehicular network in a cross layer approach. The high mobility of vehicles in vehicular communication results in the change in topology of the Vehicular Ad-hoc Network (VANET) dynamically, and the communication link between two vehicles might remain active only for short duration of time. In order for VANET to make a connection for long time and to mitigate adverse effects due to high and fixed transmission power, the proposed algorithm adapts transmission power dynamically based on estimated local traffic density. In addition to that, the prioritization of messages according to their urgency is performed for timely propagation of high priority messages to the destination region. In this paper, we incorporate the contention based MAC protocol 802.11e enhanced distributed channel access (EDCA) mechanism to implement a prioritybased vehicle-to-vehicle (V2V) communication. Simulation results show that the proposed algorithm is successful in getting better throughput with lower average end-to-end delay than the algorithm with static/default parameters.
\end{abstract}

\section{INTRODUCTION}

Vehicular Ad Hoc Networks (VANETs) are an integral part of the safety transportation systems and play a major role in intelligent transportation system. In vehicular communication, message propagation and dissemination occur with vehicle-tovehicle and/or vehicle-to-infrastructure communication. The message forwarding and propagation should be done in small amount of time. Therefore, reliability and low delay are extremely important factors for VANET applications to propagate and disseminate the message to the region of interest.

The mobility of vehicles in different traffic conditions, such as during traffic jams, accidents, traffic lights, rush hours, late night, school areas, etc., results in dynamic changes in vehicular network topology. In rural highways and during late night hours, the vehicles move with high speed, and this phenomenon results in a sparse ad hoc network. The communication link for a vehicle-to-vehicle network remains active for a short duration of time because of the highly dynamic topology of vehicular network. One way of making long time vehicle-to-vehicle connections for communication is by increasing the transmission power for sparse ad hoc networks such as in rural areas or in urban areas where the application penetration ratio is low. The high transmission power results in high interference and high network overhead in vehicular networks in highly dense traffic (e.g., areas with a high penetration ratio, urban areas, or traffic jams). Therefore in order to address these problems, dynamic adaptation of transmission power is crucial. We propose to decrease the transmission power for high vehicle density or high penetration ratio and increase the transmission power for lower vehicle density or penetration ratio.

Vehicular communication helps to reduce the number of accidents and possible deaths by propagating messages prior to such severe accidents. In order to propagate and disseminate emergency messages in a timely manner, the vehicular network for an intelligent transportation system should support message differentiation similar to service differentiation in the contention based channel access mechanism, 802.11e EDCA [1]. The priority should be assigned according to the importance of the message and time delay requirements. Messages related to accidents should propagate to target regions on time so that further congestion and possible accidents can be avoided.

Recently the adaptation of transmission power based on local density (number of vehicles per $\mathrm{km}$ ) has been proposed in [2]. However, prioritization of messages according to the urgency and the adaptation of the contention window for transmission opportunity have not been considered. In [3], Balon and Guo have proposed dynamic adaptation of the contention window according to network conditions for VANETs. They have considered the channel access time according to their urgency and delay requirements; however, they have not considered transmission power adaptation. In [4], Suthaputchakun and Ganz incorporated the 802.11e EDCA mechanism; however they considered neither adaptation of QoS parameters nor adaptation of transmission power.

Our work is motivated by the fact that the fixed transmit power and the QoS related parameters for prioritized messages do not enhance the performance for the dynamically changing topology of VANETs. Therefore the dynamic adaptation of joint transmission power and QoS parameters (e.g. contention window) in vehicle-to-vehicle communication is needed in 
order to achieve better performance according to the local density of vehicle in a cross layer approach.

The paper is organized as follows: In Section II, we present the overview of 802.11e EDCA and the problem statement. We present the dynamic adaptation of joint transmission power and contention window scheme in Section III. In Section IV, we present the proposed algorithm. The simulation set up is presented in Section V. The simulation results and discussion are presented in Section VI. Finally, the conclusion is presented in Section VII.

\section{OVERVIEW OF IEEE 802.11e EDCA AND ITS ROLE IN VANET}

The fundamental access mechanism of IEEE 802.11 is applicable in vehicular communication but does not provide any message differentiation and hence does not assign priority during dissemination of emergency messages. Moreover, VANET MAC protocols have to consider the fast changing network topology as well as the different types of messages and also have to reduce the medium access delay according to message priority. For example, it is highly desirable for emergency messages to have higher priority over other messages to get channel access.

According to [1], a QoS station implements four access categories (ACs), and there is a set of EDCA parameters associated with each AC. Those parameters include arbitration interframe space AIFS [AC] and contention window (CW) with its minimum and maximum value $\mathrm{CWmin}$ [AC] and CWmax [AC], respectively. Each AC from every station independently starts a backoff timer after detecting that the channel is idle for an AIFS [AC] interval and competes with other ACs to gain a transmission opportunity. For each AC, the backoff period is selected from a uniform distribution over $[0, \mathrm{CW}[\mathrm{AC}]]$. The $\mathrm{CW}$ size is initially assigned $\mathrm{CWmin}$ and doubles when transmission fails, up to CWmax. The smaller CWmin [AC] is, the shorter the channel access delay for the corresponding priority and hence the better chance to access the media for a given traffic condition. When an application is admitted, it will be attached with QoS parameters. If two or more backoff timers within the same station finish backoff at the same time, there will be a virtual collision. The station's internal scheduler solves the virtual collision.

Broadcasting of messages can result in the "broadcast storm" problem [9] that might result in higher delay for high priority or emergency messages. High transmission power in the region of high vehicle density results high network load in vehicular network because of broadcast and rebroadcast by large number of vehicles. Therefore, vehicle density in the region is a useful metric for adapting transmission power. Moreover, providing reliable delivery of broadcast messages in VANET introduces several challenges, such as lack of retransmission for failed broadcast transmissions.

Generally, dynamic adaptation of transmission power, based on local vehicle density, results in high throughput and timely dissemination of messages and dynamic adaptation of contention window size in EDCA gives better message differentiation. Using dynamic adaptation of transmission power along with that of the contention window combines the advantages of both methods. Hence the message propagation and dissemination occur with high throughput and low delay for priority messages. The proposed algorithm uses a cross layer approach as the algorithm adapts the transmission power (in PHY-layer) and contention window size (in MAC-layer) to enhance the performance of vehicular communication.

\section{TRANSMISSION POWER AND PRIORITIZATION OF MESSAGES}

In this section, we describe the calculation of transmission range and transmission power based on local density of vehicles and the different message categories according to their urgency.

\section{A. Transmission Range and Transmission Power}

From traffic flow theory, the transmission range is a function of the local density of vehicles, which is determined by the vehicles' movement or speed [2]. The transmission range is determined by

$T R=\min (L \times(1-K), \sqrt{(L \times \ln (L) / K})+a \times L)$

where $L$ is the length of road segment, $K$ is the estimated vehicle density, and $a$ is a traffic constant from traffic flow theory [2], [7]. Equation (1) calculates the transmission range based on the estimated local traffic density $K$. We have calculated the reachable neighbors for a given vehicle based on the method proposed in [3]. The given vehicle counts its neighboring vehicles based on the sequence number acknowledgement received from them and evaluates the $K$ value by taking the ratio of total reachable neighbors to the maximum possible number of vehicles on the given road segment.

Since traffic flow theory gives only the transmission range using equation (1), we need the proper mapping from transmission range to transmission power. Therefore we ran a separate simulation to get the transmission power corresponding to the given transmission range. The mapping results are not included in the paper because of page limit.

\section{B. Prioritization of Messages}

As discussed in section II, 802.11e EDCA has the service differentiation to provide QoS for different types of messages: voice traffic, video traffic, best effort traffic and background traffic [1]. In order to incorporate the EDCA mechanism in VANET we categorize the different messages according to their urgency and delay requirements [4] as listed in Table I.

The different access categories will have different QoS parameters associated with them. Table II gives the QoS parameters corresponding to the ACs in 802.11e EDCA. The higher the access category number, the higher the channel 
TABLE I

Message Priority [4]

\begin{tabular}{|l|l|}
\hline Priority (traffic in EDCA) & Message Type for VANET \\
\hline $\begin{array}{l}\text { Priority 1 (Voice traffic - } \\
\text { AC(3)) }\end{array}$ & $\begin{array}{l}\text { Accident message, messages from } \\
\text { emergency vehicle }\end{array}$ \\
\hline $\begin{array}{l}\text { Priority 2 (Video traffic - } \\
\text { AC(2)) }\end{array}$ & $\begin{array}{l}\text { Accident indication messages, } \\
\text { sensing vehicle tyres }\end{array}$ \\
\hline $\begin{array}{l}\text { Priority3 (Best-effort traffic } \\
-\mathrm{AC}(1))\end{array}$ & $\begin{array}{l}\text { Warning related messages, e.g. } \\
\text { work ahead, school area ahead, } \\
\text { etc. }\end{array}$ \\
\hline $\begin{array}{l}\text { Priority } 4(\text { Background } \\
\text { traffic }-\mathrm{AC}(0))\end{array}$ & $\begin{array}{l}\text { General messages e.g. periodic } \\
\text { broadcast messages }\end{array}$ \\
\hline
\end{tabular}

TABLE II

Priority SPECIFIC PARAMETERS [1]

\begin{tabular}{|c|c|c|c|}
\hline $\mathbf{A C}$ & $\mathbf{C W m i n}$ & $\mathbf{C W m a x}$ & AIFS \\
\hline 0 & CWmin & CWmax & 2 \\
\hline 1 & CWmin & CWmax & 1 \\
\hline 2 & $(\mathrm{CWmin}+1) / 2-1$ & $\mathrm{CWmin}$ & 1 \\
\hline 3 & $(\mathrm{CWmin}+1) / 4-1$ & $(\mathrm{CWmin}+1) / 2$ & 1 \\
\hline
\end{tabular}

access or transmission opportunity will be. That means the $\mathrm{CWmin}$ value for AC (3) will be the least among all ACs. The backoff counter drawn uniformly from [0, CW[AC]] will have an initial value of CWmin, implying that AC (3) will get the highest transmission opportunity over others. Each vehicle will have four different queues for each priority with a virtual collision handler to handle internal collisions.

\section{PROPOSED AlgORITHM FOR DYNAMIC ADAPTATION OF TRANSMISSION POWER AND TRANSMISSION TIME}

In the proposed algorithm, each vehicle calculates its own transmission power dynamically based on the local density in order to mitigate the adverse effects of high transmission power and to increase the duration of the communication link in case of low traffic density for inter-vehicle communication. The density is calculated using the method proposed in [3] as mentioned in Section III (A). The method does not introduce significant network overhead to identify the neighbors of the vehicle. The algorithm to calculate the transmission range according to the estimated local density of vehicles [2] is modified in this paper to set the transmission power corresponding to the calculated transmission range. Formally, the modified algorithm for the adaptation of transmission power is presented as follows:

Algorithm 1:

1. $R$ is the maximum transmission range set by default

2. INPUT: traffic constant ' $a$ ' from traffic flow theory [1], [7]

3. OUTPUT: mapped transmission power corresponding to the calculated transmission range, TR

4. $K=$ estimated local vehicle density

5. IF $(K<$ threshold 0$)$ or (Priority 1$)$ then

- Assign transmission range (TR) equal to maximum value $R$

6. OTHERWISE

- Calculate the transmission range using equation (1).

7. $E N D$

8. Assign the transmission power corresponding to calculated transmission range, $T R$.

9. STOP.

We conducted a simulation to find the transmission range in meters corresponding to the transmission power in $\mathrm{dBm}$. We made a suitable mapping of transmission power and transmission range so that the algorithm could take the suitable value for transmission power corresponding to the calculated value of transmission range.

The priority 1 message, being the highest priority and having a requirement of low delay, should propagate on time in a single hop (if possible) within the maximum transmission range. In order to incorporate this rule, the algorithm always assigns the maximum transmission range for the vehicle that carries priority 1 messages.

To support message differentiation for different types of messages listed in Table I, the algorithm has to choose the QoS parameters considering that the higher priority message holding vehicles should not get a chance to be greedy, and at the same time, the higher priority messages should not be waiting for a long time to get a transmit opportunity. For higher priority messages, waiting for a long time to get channel access might result in a delay in message propagation. Therefore, in order to mitigate the adverse effects in both cases, the dynamic adaptation of QoS parameters, in particular $\mathrm{CW}$ values, for different access categories is essential since the backoff counter value is obtained uniformly from $[0$, $\mathrm{CW}[\mathrm{AC}]]$ and the initial $\mathrm{CW}$ value is $\mathrm{CWmin}$. Therefore the dynamic adaptation of $\mathrm{CW}$ causes the change in back-off counter so that timely transmission of messages happens according to the local density. In order to change the contention window size dynamically, we have considered the collision rate in the channel and the number of backoff times for all messages as triggering metrics. 
The formal algorithm to adapt the $\mathrm{CW}$ [AC] value that gives the transmission opportunity to access the channel for corresponding messages or ACs is as follows:

Algorithm 2:

\section{IF collision occurred $>$ threshold 1}

- Increase the corresponding $C W[A C]$ values

2. ELSE IF collision occurred $<$ threshold1

- Decrease the corresponding $C W[A C]$ values

\section{OTHERWISE}

\section{- Maintain corresponding $C W[A C]$ values}

4. STOP.

The proper choice of threshold value also plays an important role for the algorithm. Algorithm 1 adapts the transmission range and eventually the transmission power, whereas algorithm 2 adapts the corresponding $\mathrm{CW}$ values for all messages, hence the transmission opportunity or time to get channel access. In order to adapt the transmission power and the contention window (transmission time) dynamically, each vehicle runs both algorithms in a periodic manner so that the proper tuning of those values occurs according to the local density of vehicles and the network conditions.

\section{Simulation SetuP}

In this section, we present the simulation setup to evaluate the performance of the proposed scheme. The simulation is performed using ns-2 [5] with added support for EDCA developed in [8]. Two scenarios are considered: one with the default scheme (having all values fixed for all parameters) and the other with the proposed scheme (having dynamic adaptation of both transmission power and $\mathrm{CW}$ value). We have compared the overall throughput and the average end-toend delay for all messages and for the highest priority message for 150 mobile nodes acting as vehicles.

For both simulations, we use the Poisson data generation with a packet size of 512 Bytes and rate of 10 packets per second. As we have mentioned in Section-II, each priority message will have the QoS parameters and corresponding default values as listed in Table II.

The value of the traffic constant used in equation (1) is assumed to be $a=0.25$ as per traffic flow theory [2], and the length of road segment $L$ is taken as $500 \mathrm{~m}$ ( 0.311 mile). The vehicle calculates its local vehicle density $K$ periodically using the approach mentioned in Section IV.

In both simulation scenarios, we have assumed that each vehicle maintains the safety distance. Using microscopic traffic Intelligent Driver Model (IDM) [6], we have simulated a $16094 \mathrm{~m}$ (10 mile) urban map with one lane in each direction, with an initial vehicle speed in the range of $12-30$ $\mathrm{m} / \mathrm{s}(30-68 \mathrm{mph})$ which can be increased or decreased to maintain the safety distance.
In order to incorporate the EDCA mechanism in VANET using ns-2, we have mapped the suitable messages with the corresponding service differentiated EDCA access categories as listed in Table I.

We have set vehicles to receive all types of messages. But we have assigned 150 vehicles randomly to act as sources for different messages. In the simulation, we have generated the voice traffic as a message related to an accident, video conferencing traffic as a message indicating possible accident, HTTP browsing as warning related messages, and FTP traffic as regular periodic messages.

\section{SimUlation RESUlTS AND DISCUSSION}

In this section, we present the simulation results and discussion in order to corroborate our findings. We study the performance of proposed scheme using overall throughput and average end-to-end delay for messages. We compared the results obtained from proposed scheme with those of fixed parameter scheme.

We simulated both scenarios for 300 s and collected the global statistics for overall throughput and average end-to-end delay.

Figure 1 shows that the overall throughput is higher for proposed scheme than that of default scheme. Initially the throughput value is same for both schemes since the vehicles might not be able to detect their neighbors (vehicle density) at first and will not be able to tune the parameters. The proposed algorithm decreases the transmission power when the vehicle density is higher than the threshold value and increases the transmission power when the local vehicle density is lower. The higher priority messages get higher opportunity for transmission, and the adjustment in transmission power and QoS parameter makes low network overhead because of the lower range of broadcast and rebroadcast of messages within the local region. As a result the overall throughput of the proposed scheme is higher than that of default scheme.

We observe that the average end-to-end delay is decreasing in both scenarios and the delay is lower for proposed scheme than that of default scheme as shown in Figure 2. Individual vehicles adapt the transmission power and CW values. Since the adaptation of the $\mathrm{CW}$ values determines the channel access opportunity according to collision rate in the network, i.e., when the network collision rate is higher individual vehicles increase the $\mathrm{CW}$ value (maintaining EDCA hierarchy) associated with messages so that they get lower opportunity for channel access, and when the network has a low collision rate, the individual vehicles start to decrease the $\mathrm{CW}$ values (maintaining the EDCA hierarchy) to get higher opportunity for channel access. The adaptation of the $\mathrm{CW}$ value protects the network from a greedy transmitter and also protects higher priority messages from waiting a long time to get channel access. As a result of adaptation, the proposed scheme reduces end-to-end delay by reducing possible message collisions in the network producing timely dissemination of messages. 


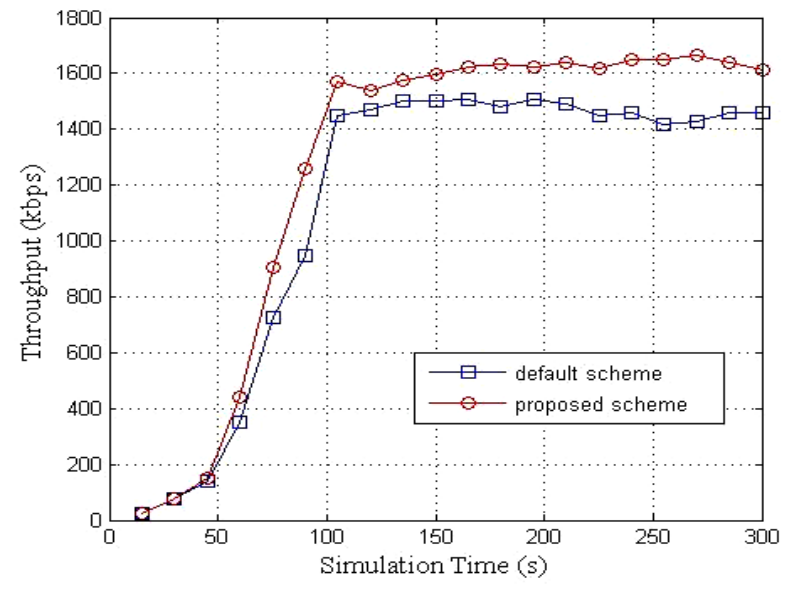

Fig. 1 Overall throughput vs. simulation time

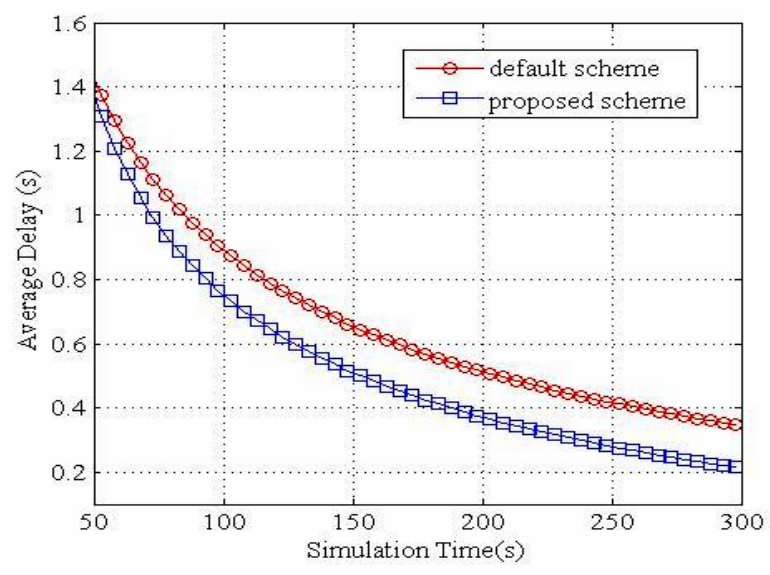

Fig. 2 Average end-to-end delay for all traffic vs. simulation time

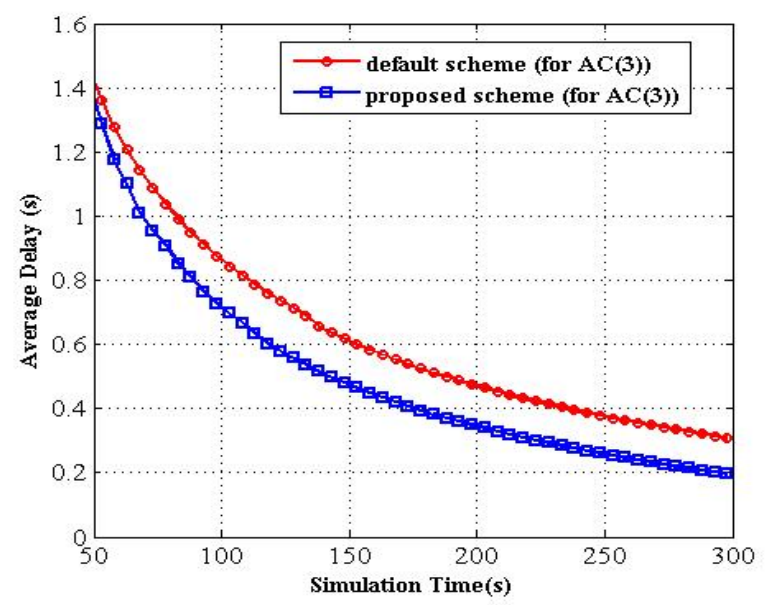

Fig. 3 Average end-to-end delay for highest priority traffic vs. simulation time

In Figure 3, we plot the average end-to-end delay only for the highest priority message. The delay is higher in the case of the default scheme than that of proposed scheme. In the proposed scheme, because of the proper tuning of $\mathrm{CW}$ and transmission power, the highest priority messages will have higher chances of channel access and the message can disseminate towards the destination region on time. We also note that the delay for the highest priority message in Figure 3 is slightly less in compared to corresponding overall average end-to-end delay in Figure 2. We note that this happened because most of the vehicles were carrying highest priority, $\mathrm{AC}(3)$, messages in our simulation.

\section{CONCLUSIONS}

In this paper, we presented the algorithms (algorithm 1 and 2) for dynamic adaptation of joint transmission power and contention window according to the vehicle density and network traffic conditions in a cross layer approach to improve the performance. The vehicle evaluates the local density of vehicles and calculates the transmission range and corresponding power based on the transmission range. We incorporated the medium access mechanism of IEEE 802.11e EDCA in VANET to set priority for different messages according to their urgency. With the help of ns-2, dynamic adaptation of QoS parameters $(\mathrm{CW})$ along with transmission power was performed to improve the performance of the vehicular communication. Simulation results have shown that the better throughput is obtained with lower delay for high priority messages by adjusting the QoS parameters according to the network conditions and adjusting the transmission power based on local density. Thus the proposed scheme enhances the overall performance with high throughput and less delay.

\section{ACKNOWLEDGEMENT}

This work was supported in part by the National Science Foundation under Grant CNS-0721586.

\section{REFERENCES}

[1] Part 11: Wireless LAN Medium Access Control (MAC) and Physical Layer (PHY) specifications Amendment 8: Medium Access Control (MAC) Quality of Service Enhancements, IEEE Std. 802.11e, 2005.

[2] M. M. Artimy, W. Robertson, and W. J. Phillips, "Assignment of dynamic transmission range based on estimation of vehicle density," VANET'05, Cologne, Germany, Pages: 40 - 48, September 2005.

[3] N. Balon and J. Guo, "Increasing Broadcast Reliability in Vehicular Ad Hoc Networks," VANET'06, Los Angeles, California, Pages: 104 - 105, 2006

[4] C. Suthaputchakun and A. Ganz, "Priority Based Inter-Vehicle Communication in Vehicular Ad-Hoc Networks using IEEE 802.11e," VTC2007-Spring, IEEE 65th, Pages: 2595-2599, Year: Apr. 2007.

[5] Network simulator 2 (ns-2) http:// www.isi.edu/nsnam/ns

[6] M. Treiber, A. Hennecke, and D. Helbing, "Congested traffic states in empirical observations and microscopic simulations," Phys. Rev. E, vol. 62, no. 2, pp. 1805-1824, Aug 2000.

[7] R. P. Roess, E. S. Prassas, and W. R. McShane. Traffic Engineering. Pearson Prentice Hall, Third edition, Erewhon, NC, 2004.

[8] S. Wiethölter and C. Hoene: "Design and Verification of an IEEE 802.11e EDCF Simulation Model in ns-2.26", TKN-03-19 Technical Report Series, November 2003.

[9] S.-Y. Ni, Y.-C. Tseng, Y.-S. Chen, and J.-P. Sheu, "The Broadcast Storm Problem in a Mobile Ad Hoc Network," in Proceedings of the fifth annual ACM/IEEE international conference on Mobile computing and networking, , pp. 151-162, August 1999. 